Inderjeet Parmar/Michael Cox (Hrsg.), Soft Power and US Foreign Policy: Theoretical, Historical and Contemporary Perspectives, New York u.a. (Routledge) 2010.

Das zunehmend negative Image der USamerikanischen Außenpolitik, insbesondere nach dem Irakkrieg und dem sogenannten war on terror, rückte den von Joseph Nye geprägten Begriff der Soft Power erneut in den Fokus von Wissenschaft und praktischer Politik. Das vorliegende Werk beinhaltet neben einer neuen Stellungnahme von Nye eine kritische Auseinandersetzung mit dem Konzept aus theoretischem, historischem und institutionellem Blickwinkel.

Soft Power bedeutet das Erreichen außenpolitischer Ziele durch Anziehungs- (attraction) und Überzeugungskraft (persuasion), im Gegensatz zu Zwang (coercion) und Gewalt (force). Diese Art der Macht gründet größtenteils auf der Kultur, den politisches Idealen und den Politikinhalten eines Landes, wohingegen Hard Power auf militärischen und ökonomischen Größen beruht.

Grundlage für das von Parmar und Cox herausgegebene Werk ist ein von der Research Group on American Power veranstaltetes Symposium im Mai 2008. Wie die Veranstaltung gliedert sich auch die hier besprochene Publikation in sechs Kapitel. Ausgehend von einer Darlegung ihrer eigenen Sichtweisen, kritisieren und reflektieren die Autoren in vier Themengebieten mit insgesamt elf Aufsätzen die Ansichten Nyes, bevor dieser am Schluss des Sammelbands zu den Kommentaren Stellung bezieht. Dieser letzte Abschnitt stellt die innovativste Passage des Sammelbands dar und wird aus diesem Grunde nach einem Überblick über die restlichen Kapitel eingehender betrachtet.

$\mathrm{Zu}$ Beginn des Bandes legt Nye seine Sichtweisen zu Soft, Hard und Smart Power dar, bewertet die Außenpolitik Bushs und gibt Empfehlungen für die aktuelle Regierung unter Barack Obama. Daran anschließend folgen drei Aufsätze mit theoretischen und methodologischen Kritiken an Nyes Konzept der Soft Power. Vor allem der Beitrag von Leonardo Ramos und Geraldo Zahran über die Verbindung des Soft-Power-Konzepts zu Antonio Gramscis Verständnis von
Macht als Konsens ist erwähnenswert und findet dementsprechend auch besondere Aufmerksamkeit in der späteren Stellungnahme Nyes. Auf die historische Dimension bzw. die Verbindung von $\mathrm{Pu}$ blic Diplomacy und Soft Power fokussieren je zur Hälfte die nächsten sechs Beiträge des Bandes. Abschließend werden die Europäische Union und China in Bezug auf ihre Soft-Power-Performance betrachtet. Einen außerordentlichen Beitrag leistet dabei Christopher Hill, der das außenpolitische Verhalten der EU im Zeitraffer auf seine Soft-Power-Strategie hin untersucht.

Im Schlusskapitel wird eine ganze Bandbreite von Themen, mal mehr und mal weniger umfassend von Nye behandelt. Er baut zumeist auf den Kritiken aus dem Sammelband auf, um im Weiteren sein Konzept zu verteidigen. So gehen vor allem die Behandlung der Problematik der Definition von Macht und die Auseinandersetzung mit Anfechtungen gegenüber den Ressourcen von Soft Power über die zuvor vorgebrachten Punkte hinaus. Von den zumeist punktgenauen und überzeugenden Stellungnahmen sind vor allem jene erleuchtend, die sich mit der Kritik einer zu einfachen und inkonsistenten Begriffsbestimmung von Macht in den Werken Nyes befassen. Wie der Begriff der Macht definiert wird, hängt Nye zufolge von den jeweiligen Interessen und Werten ab. Da sich diese von 1990 bis 2008 immer wieder verschoben haben, so Nye, habe sich auch seine Verwendung des Machtbegriffs gewandelt. In Bezug auf den Unterschied zwischen Machtausübung und Machtquellen erläutert Nye diese zuerst abstrakt, bevor er eine mögliche Verwechslung zwischen beiden mit Hilfe von Beispielen verdeutlicht. Im Weiteren verwirft Nye den Vergleich zwischen dem Konzept der Soft Power und Gramscis Diskurshegemonie mit einem Verweis darauf, dass die von den USA vertretenen Werte nicht universal seien und unterschiedliche Auffassungen der Staaten in Bezug auf Wertesysteme bestünden.

Etwas kürzer und dennoch umfassend werden Thesen zu einem Niedergang der USA und der Reduzierung des SmartPower-Konzepts auf die Qualität eines Slogans behandelt. Nye bleibt in Bezug auf die US-amerikanischen Weltmachtstellung seiner bereits zuvor bekannten und auf Fareed Zakaria zurückgehenden Argumentation treu, nämlich dass die aktuelle Situation weniger eine des American decline, als vielmehr eine des Aufstiegs anderer Nationen sei. Darüber hinaus stellt er den viel beschworenen zukünftigen Niedergang der USA generell in Frage. Der Verlust an Soft Power während des Vietnamkriegs und die darauffolgende Verbesserung des Images durch die geänderte Außenpolitik zeigen seiner Auffassung nach, dass Barack Obama durchaus eine Kursänderung vornehmen könne. Dem Vorwurf in Bezug auf Smart Power entgegnet Nye, dass die Elemente des Konzepts - eine gute Balance zwischen Soft und Hard Power - zwar zur „Plakatierung“ missbraucht werden könnten, die deskriptive Dimension allerdings einen Rahmen für die Entwicklung von effektiven Strategien in den verschiedensten Kontexten biete.

Die Beiträge des Sammelbandes sind allesamt von hoher Qualität, wenn bisweilen auch alter Wein in neuen Schläuchen geboten wird. Nyes Beiträge machen den Band - was die übrigen Autoren anerkennen - einzigartig. Empfehlenswert ist eine Lektüre des Werkes vor allem für Fachleute im Bereich der Internationalen Beziehungen; viele der Aufsätze sind jedoch auch für allgemein politisch interessierte Leser geeignet.

Sascha Knöpfel

Daniel Marc Segesser, Recht statt Rache oder Rache durch Recht? Die Ahndung von Kriegsverbrechen in der internationalen wissenschaftlichen Debatte 1872-1945 (Krieg in der Geschichte, Bd. 38), Paderborn (Ferdinand Schöningh) 2010.

Mit der akribisch gearbeiteten Studie liegt die umfassendste historische Darstellung des Problemkomplexes Ahndung von Kriegsverbrechen in deutscher Sprache vor. Der Autor hat an die hundert einschlägige Fachzeitschriften und eine solche Fülle an Literatur ausgewertet, sodass auf absehbare Zeit wohl keine weitere Arbeit dieses Umfangs und mit einem solchen Detailreichtum erscheinen wird.

Der Autor stellt die juristische und rechtspolitische Diskussion um Kriegsverbrechen und ihre Ahndung in den jeweiligen historischen und politischen 
Zusammenhang und macht so nachvollziehbar, wie die internationale Diskussion von den historischen Ereignissen und politischen Auseinandersetzungen mit geprägt wurde.

Die Studie folgt der historischen Entwicklung in fünf Kapiteln. Zunächst wird die Debatte von 1872 bis 1908 vor dem Hintergrund der Kriegsverbrechen im deutsch-französischen Krieg, im amerikanischen Bürgerkrieg sowie im Burenkrieg und im russisch-japanischen Krieg nachgezeichnet. Besonderes Gewicht wird dabei auf die Diskussion während der Haager Friedenskonferenzen von 1899 und 1907 gelegt. Im Mittelpunkt stehen die Bemühungen um eine Zivilisierung oder Domestizierung des Krieges durch eine Erweiterung bzw. Präzisierung des ius in bello.

Im zweiten historischen Abschnitt der Arbeit werden die politischen und fachjuristischen Diskussionen angesichts der Balkankriege und des Ersten Weltkriegs referiert. In diesem Kapitel, in dem auch die allmähliche Durchsetzung des Begriffs Kriegsverbrechen, der zuvor kaum verwendet worden ist, nachgezeichnet wird, werden die erfolglosen Bemühungen um eine Strafgerichtsbarkeit aufgezeigt, die in Deutschland mit der Farce der Leipziger Kriegsverbrecherprozesse endete.

Der Erste Weltkrieg hatte jedoch Anlass gegeben, das Problem der Kriegsverbrechen auch über den Krieg hinaus auf der Tagesordnung zu halten. Neue Akteure traten in die Diskussion ein: die Interparlamentarische Union und der Völkerbund. Die Bemühungen um einen internationalen Strafgerichtshof wurden intensiver, neue Verträge mit Konsequenzen für Kriegsverbrechen wurden geschlossen: der Briand-Kellogg-Pakt und die Konvention zur Bekämpfung des Terrorismus.

Überrollt wurden alle diese Diskussionen und Bemühungen durch den Zweiten Weltkrieg. Nach dem Krieg hatte die Diskussion um Kriegsverbrechen aber konkrete politische Folgen. Die United Nations War Crime Commission nahm ihre Arbeit auf und die Londoner Charta zur Schaffung eines internationalen Militärtribunals führte zu den Nürnberger Kriegsverbrecherprozessen. Die juristische Aufarbeitung und Aburteilung von Kriegsverbrechern setzte sich gegen summarische Erschießungen verantwortlicher Politiker und anderer Kriegsverbrecher durch.

Der Buchtitel verweist auf das Schlusskapitel des Buches, in dem die Frage der Ahndung von Kriegsverbrechen explizit diskutiert wird. Hier werden die Zielvorstellungen der Initiatoren des 19. Jahrhunderts mit den konkreten Ergebnissen des Nürnberger Tribunals verglichen. Diese Diskussion hat etwas gewollt Schulmäßiges an sich.

Das Buch bietet der Leserin bzw. dem Leser eine Fülle von Informationen und macht es ihr bzw. ihm nicht immer leicht, den Überblick zu behalten. Allerdings ist das umfängliche Register eine gute Hilfe, Querverbindungen zu ziehen und Personen und Sachverhalte miteinander zu verknüpfen. Diese Habilitationsschrift wird lange Gültigkeit behalten und kann zugleich als ein Handbuch zur rechtshistorischen Entwicklung des Themas genutzt werden.

\section{Martin Kutz}

Anne Wildfang, Terrorismus - Definition, Struktur, Dynamik, Berlin (Duncker \& Humblot) 2010.

Terrorismus hat seit 2001 in der öffentlichen und wissenschaftlichen Diskussion enorm an Bedeutung gewonnen, vieles wurde seitdem als terroristisches Potenzial und terroristische Bedrohung betrachtet. Mit der Frage, was genau Terrorismus ist, beschäftigt sich Anne Wildfang in ihrer kriminologischen Dissertation. Ihr erklärtes Ziel ist es, „zu einer angemessenen Reaktion auf die Bedrohung der inneren und äußeren Sicherheit durch terroristische Gruppen beizutragen" (S. V). Zudem möchte sie die von ihr kritisierte grundlegende kriminologische „Forschungslücke durch eine Eingrenzung der Phänomenologie des Terrorismus sowie einen kriminologischen, theoretischen Zugang [...] schließen" (S. 4). Hierzu definiert sie zunächst ihren Untersuchungsgegenstand, geht dann auf die verschiedenen Erscheinungsformen des Terrorismus ein, um im Anschluss zu analysieren, aus welcher Motivation heraus sich Individuen terroristischen Gruppen anschließen und wie diese Gruppen funktionieren.
Die Autorin bemüht sich um eine klare Abgrenzung zu anderen, dem Terrorismus verwandten Phänomenen, wie beispielsweise Staatsterrorismus, Extremismus, Befreiungsbewegungen, Guerilla oder organisierte Kriminalität, verweist aber darauf, dass dies keine erschöpfende Aufzählung ist. Aus ihrer kriminologischen Sicht ist Terrorismus mit internationaler Kriminalität vergleichbar. Wenn Staaten Terroristen als Kriegspartei statt als Kriminelle betrachten und die entsprechenden Maßnahmen ergreifen, sei dies keine angemessene Reaktion. Kritisch sieht sie im Zusammenhang mit der Strafverfolgung die am Strafrecht der BRD (§§ 129ff.) beispielhaft dargelegte Wandlung „vom Ultima-Ratio-Prinzip hin zum Risikostrafrecht“ (S. 29) und plädiert für einen innovativeren Umgang mit Terroristen. Als Abschluss des ersten Teils der Studie formuliert sie in ihrer Arbeitsdefinition folgende, sehr eng gefasste, Kriterien für Terrorismus: Es handelt sich um ein „politisches, kriminelles, kollektives Gewaltphänomen“, das sich „gegen den Status quo einer Gesellschaft" richtet und dabei in Form von „kleinen, organisierten Gruppe[n] [...] aus dem Untergrund heraus“ versucht, ein „Klima der Angst und Ohnmacht“ (S. 71) zu erzeugen. Die Selbstrechtfertigung und Formulierung des Ziels erfolgt über die jeweilige Ideologie. Als zentral betrachtet Wildfang die Nutzung der Medien durch Terroristen, wobei wiederum die Medien terroristischen Handlungen bereitwillig die gewünschte Aufmerksamkeit in Form von Schlagzeilen schenken.

Nach einer knappen Bestimmung der Begriffe Gruppe, Organisation, Masse und System sowie der Betonung der Bedeutung von Kommunikation, konzentriert Wildfang sich insbesondere auf die Struktur terroristischer Gruppen. So können terroristische Gruppen zwar durchaus auch als Organisationen aufgefasst werden, der Gruppenaspekt bleibt aber zentral. Entscheidungen werden meist auf $\mathrm{Ba}$ sis hierarchischer Prozesse gefällt, auch Netzwerkstrukturen können jedoch eine Rolle spielen.

Anschließend untersucht Wildfang, mittels welcher Theorien erklärt werden kann, warum sich Individuen terroristischen Gruppen anschließen. Nach einer ausführlichen Erläuterung, warum herkömmliche Kriminalitätstheorien 
dies nicht hinreichend erklären können, stützt Wildfang ihre Analyse auf konstruktivistische Ansätze und insbesondere die Theorie der sozialen Identität sowie das Konzept der Gruppendynamik. Für einen Gruppeneintritt ist demnach sowohl die Selbst- als auch die Fremdwahrnehmung des Individuums ausschlaggebend, das durch seine Mitgliedschaft persönliche Stabilität und Sicherheit erreichen will. Je ähnlicher ihm die Gruppe ist, desto wahrscheinlicher wird es sich ihr anschließen. Eine Selbstaufwertung von Individuum und Gruppe wird insbesondere durch Abgrenzung vorgenommen, aber auch durch Bezug auf bestimmte Symboliken und kognitive Strategien. Wildfang zufolge werden „die Inhalte der terroristischen, sozialen Identität immer mehr zu Inhalten der persönlichen Identität“ (S. 163). Zwar ist eine Ideologie für die soziale Identität der terroristischen Gruppe von Bedeutung, die konkreten Inhalte der Ideologie sind dabei jedoch nicht ausschlaggebend.

Wildfang zufolge dient jegliches Handeln der Gruppe dem Selbsterhalt und soll sie gegen den von ihr konstruierten Feind schützen. Letzten Endes wird „aus dem eingebildeten Wahn, der Selbstschutzmaßnahme, Wirklichkeit“ (S. 206), wenn der angegriffene „Feind“ reagiert und gegen die Gruppe agiert. Wildfang mahnt an, diesen Aspekt im Rahmen der Terrorismusbekämpfung stets im Auge zu behalten und „die schwierige Balance zwischen Effektivität und möglichem Sympathieverlust“ (S. 235) zu beachten. Zudem müsse in Rechnung gestellt werden, dass „der Ausstieg neben den sozialpsychologisch bedingten Hemmungen oft faktisch erheblich erschwert" (S. 242) wird, da das Individuum sich nicht selbst eingestehen möchte, dass die Gruppe möglicherweise nicht die sozial erwünschte richtige Identität bereithält. Zudem wird von der Gruppe selbst in der Regel enormer Druck aus Angst vor Verrat und Entdeckung aufgebaut.

Die Arbeit Wildfangs stellt einen wichtigen Schritt kriminologischer Forschung im Bereich Terrorismus dar. Politische Lösungsansätze kommen allerdings etwas zu kurz. Zwar wird darauf eingegangen, dass mehr Aufklärung betrieben, „nicht-terroristische[r], stabile[r], positiv bewertete[r] soziale[r] Identitäten “ (S. 263) gefördert und „Identitätsalterna- tiven“ (S. 269) angeboten werden sollten, jedoch kommt es zu keiner Konkretisierung dieser Vorschläge.

Daniela Kost

Thomas Weitin (Hrsg.), Wahrheit und Gewalt. Der Diskurs der Folter in Europa und den USA, Bielefeld (transcript Verlag) 2010.

„Worüber redet man, wenn man von Folter spricht?“(S. 11), so lautet die zentrale Frage dieses Sammelbandes von Thomas Weitin. In zwölf Beiträgen von Experten unterschiedlicher Fachrichtungen wird versucht, die vielen Gesichter von Gewalt, meist in Form von Folter, aufzuzeigen. Dies geschieht sowohl im historischen Kontext als auch aus gegenwärtiger Perspektive. Dabei, so das Urteil des Herausgebers, zeigen sich eine Reihe von Kontinuitäten im Vergleich zwischen der historischen Debatte über Folter und der aktuellen Diskussion.

Der Band ist in drei Kapitel unterteilt. Im ersten Kapitel geht es um Grundlagen und Grundfragen in Bezug auf den Begriff der Folter. Es wird die Bedeutung der Menschenwürde in der Bundesrepublik Deutschland veranschaulicht und ein Überblick über den Wertewandel in Bezug auf die Folter gegeben.

Der zweite Teil behandelt den Diskurs über Folter in Europa und befasst sich mit der Verbindung von Folter und Märtyrertum, der Folter in historischen Strafprozessen und der Darstellung der Folter. Dabei geht es immer um die historische Entwicklung, im engen Zusammenhang auch mit Veränderungen der Darstellung der Folter in Literatur und Film.

Die USA bilden den Fokus des dritten Abschnitts des Buches. In den Beiträgen geht es zunächst um die Geschichte des „Lynching“ und der Todesstrafe in den USA; es folgt ein Beitrag über die Geschichte des Lügendetektors. Die beiden letzten Beiträge befassen sich mit den Vorfällen in Guantanamo und Abu Ghraib und geben einen Einblick in unterschiedliche Standpunkte zum Umgang mit Terrorverdächtigen. Die Begriffe Scham und Bestialität spielen bei der Bewertung und Analyse der Vorfälle in den Gefängnissen eine zentrale Rolle.
Lutz Ellrich zeigt in seinem Beitrag „Was spricht für die Folter?" zunächst die historisch wechselnde Bedeutung von Wahrheit bis hin zur Gegenwart auf. Obwohl Gewalt schon früh als Mittel zur Wahrheitsfindung angewendet wurde und bisweilen auch religiös, unter anderem im Christentum, verankert ist, kam es schon früh zu einer kritischen Auseinandersetzung mit ihr. Bereits im Altertum sind in Bezug zu der Frage des Nutzens von Informationen, die unter Folter gewonnen wurden, Bedenken geäußert worden. Doch macht der Autor deutlich, dass diese Bedenken sich historisch nicht immer durchgehalten haben und sieht gegenwärtig eine steigende Akzeptanz der „wahrheitsgenerierenden Potenziale“, die der Folter zugeschrieben werden (S. 49).

Am Ende geht der Autor auf das Für und Wider von Folter ein. Er ist der Meinung, dass hierbei keine genaue Positionierung möglich ist. Auch derjenige, der Folter grundsätzlich als nicht „richtig“ und nicht „gerecht“ ansieht, könnte zu einer abweichenden Bewertung kommen, wenn es zum Beispiel um das eigene Kind geht (S. 59). Durch Ellrichs detaillierten Überblick über die Anwendung der Folter als Instrument zur Wahrheitsfindung in Vergangenheit und Gegenwart wird deutlich, dass man sich unter ganz unterschiedlichen Gesichtspunkten mit diesem Problem auseinandersetzen und zu abweichenden Bewertungen dieses Instruments gelangen kann.

Arno Meteling setzt sich in seinem Beitrag Folterszenen mit der Darstellung der Folter in Film und Fernsehen auseinander. Dabei stellt er heraus, dass es je nach Genre starke Unterschiede in der Darstellung gibt. Er differenziert zunächst in unterschiedliche Legitimierungsformen der Folter im Film. Den Schwerpunkt legt er auf die Darstellung der Folter in den sogenannten Splatterfilmen. Dort findet eine vollständige Ablösung der Folter vom engeren Ziel der Wahrheitsfindung statt. Das Leiden des Gefolterten soll möglichst eindrücklich in Szene gesetzt werden, während die Motivation des Folterers zumeist unbekannt bleibt. Die Entwicklung hin zu dieser Art von Film wird mit der Schaulust des Publikums erklärt und mit historischen Spektakeln wie den Gladiatorenspielen der Antike verglichen. Der Beitrag bietet insgesamt einen gu- 
ten Überblick über die unterschiedliche Darstellung der Folter im Film und der filmischen Effekttechniken.

Klaus Mladek setzt sich im Kapitel Folter und Scham mit den Vorfällen in Guantanamo und Abu Ghraib auseinander. Zunächst bewertet er den Einfluss der Obama-Administration auf die ,zentralen politischen Handlungsparadigmen der USA“. Diese Paradigmen haben sich unter Obama nicht gewandelt. Mladek ist der Meinung, dass beispielsweise der „Triumph des Sicherheitsdispositivs“ (S. 245) in Verbindung mit ethisch-religiösen Wertargumenten in den USA alternative Handlungsoptionen ausschließt.

Schließlich thematisiert der Autor den Umgang mit den Gefangenen und die Frage der Scham. Die Art der Demütigungen, die die Verdächtigen erfahren haben, werden hier eindrücklich illustriert. Ein Kommandeur in Guantanamo hat den Umgang mit den Gefangenen sehr unverblümt beschrieben: „You have to treat the prisoners like dogs" (S. 251). Mladek zufolge ist durch diese Vorfälle Scham zu einem globalen Phänomen geworden. Die Fotos von Abu Ghraib seien „Zeugnisse einer Schamlosigkeit, allerdings zur Schau getragen als der Stolz der Gerechten“ (S. 254).

Insgesamt bietet das Buch differenzierte und kritische Standpunkte zur Anwendung der Folter. Es zeigt, dass es zwar durchaus Veränderungen bei der Gewaltanwendung etwa im Rahmen der Strafverfolgung gab, aber keineswegs ein „Ende der Folter“ absehbar ist. Die Darstellung des Diskurses über die Folter erfolgt sehr detailliert, zahlreiche Illustrationen und eine Fülle von Zitaten regen den Leser bzw. die Leserin zu einer vertiefenden Auseinandersetzung mit der Thematik an.

Leah Klees

Martin H. W. Möllers/Robert Chr. van Ooyen (Hrsg.), Jahrbuch Öffentliche Sicherheit 2008/2009, Frankfurt a. M. (Verlag für Polizeiwissenschaft) 2009.

Mit seiner vierten Ausgabe hat sich das Jahrbuch Öffentliche Sicherheit (JBÖS) Anerkennung als bedeutender Beitrag zur Erörterung demokratiepolitischer und sicherheitsrelevanter Fragen in Politik und Rechtswissenschaft verdient.
Das JBÖS 2008/2009 ist ein umfangreicher Sammelband, der insgesamt 47 Beiträge, unterteilt in sechs Kategorien, enthält. Sie bieten einen umfassenden Überblick über aktuelle Probleme und Diskussionsfelder der Öffentlichen Sicherheit. Behandelt werden die Themenkomplexe: Theorie der Sicherheit und Methoden der Analyse, Wehrhafte Demokratie, Extremismus/Radikalismus, Öffentliche Sicherheit in Deutschland, Europäische Sicherheitsarchitektur und Internationale Sicherheit. Mit „Theorie der Sicherheit und Methoden der Analyse“ und „Wehrhafte Demokratie“ sind zwei neue Themenfelder eingeführt worden. Auch heikle Themen, wie das Attentat auf den Passauer Polizeipräsidenten als Beispiel für aktuelle Entwicklungen im (Rechts-)Extremismus kommen zur Sprache, ebenso wie der „HeiligendammBeschluss“, der zur Verdeutlichung der Erosion des Rechts auf Versammlungsfreiheit herangezogen wird.

Die behandelten Themen wirken trotz der breiten Streuung nicht beliebig und spannen einen Bogen von theoretischen Reflexionen über den Begriff Sicherheit bis zu Beiträgen zur Ideologie des Islamismus und zu Erörterungen über die Rolle und Rechtsprechung des Bundesverfassungsgerichts zu den Grundrechten. Die bemerkenswerte thematische Diversität der Beiträge ist keineswegs das einzig auffallende Element des Jahrbuchs. Die Heterogenität der im Jahrbuch vertretenen Positionen ist ebenfalls erwähnenswert. Der Sammelband gibt keiner bestimmten politischen Position den Vorzug und beschränkt sich nicht auf eine einzige wissenschaftliche Theorie bzw. einen methodischen Ansatz.

An dieser Stelle soll ein Beitrag aus der neu eingeführten Kategorie „Theorie der Sicherheit und Methoden der Analyse“ kurz vorgestellt werden. Dieser Beitrag wurde aufgrund der Tatsache ausgewählt, dass er sich zum einen in einer der beiden neuen Kategorien verorten lässt und zum anderen, weil hier die thematisch angelegte Breite des JBÖS deutlich wird, da der Artikel das Thema Sicherheit weniger von einer politischen als vielmehr von einer soziologischen Perspektive aus beleuchtet.

Rüdiger Voigt betrachtet in seinem Beitrag zur „Sicherheit in der ,Risikogesellschaft “ - Wir Bürger als Sicherheitsrisiko?"
Rechtsstaat, Sozialstaat und Demokratie aus der Perspektive der Risikogesellschaft. Hierbei unterteilt Voigt seine Analyse in „das Zerbrechen der Moderne“, „neue globale Risiken“, „Gegenmaßnahmen“, „den Staatenschutz“, „Biopolitik“, „neue Instrumente“ und „ein Zukunftsszenario“. Im Zentrum des Artikels stehen dabei drei historische Geschehnisse: Der Reaktorunfall von Tschernobyl am 26. April 1986, die Terroranschläge vom 11. September 2001 und die Razzien in Berlin am 9. Mai 2007, die im Vorfeld der geplanten Demonstrationen gegen den G8-Gipfel in Heiligendamm durchgeführt wurden.

Im ersten Abschnitt seines Beitrags nimmt Voigt die von Ulrich Beck in seinem Buch „Risikogesellschaft“ gewonnene Erkenntnis eines Bruchs innerhalb der Moderne auf, die den Wechsel von der Logik der Reichtumsverteilung hin zur Logik der Risikoverteilung beschreibt. Er verdeutlicht dies an dem Unglück von Tschernobyl, das die globalen Auswirkungen eines atomaren Unfalls aufgezeigt hat. Nicht nur die Folgen atomarer Unfälle, sondern auch der saure Regen oder gar die Auswirkungen eines Atomkriegs machen nicht vor nationalen Grenzen halt. Selbst durch hohe private Aufwendungen lässt sich kein umfassender Schutz kaufen.

Als Beispiel für ein weiteres globales Risiko führt Voigt den Terrorismus an. Auch die Supermacht USA ist verwundbar geworden und der Anschlag auf das World Trade Center hat der (westlichen) Welt ihre relative Schutzlosigkeit, also einen Mangel an Sicherheit, vor Augen geführt. Der Autor kommt zu der Schlussfolgerung, dass der Staat heute nicht mehr originär Schutzstaat ist, wie von Hobbes im 17. Jahrhundert postuliert, sondern vielmehr durch drei Staatszielbestimmungen charakterisiert wird: Das Rechtsstaatsprinzip, das Sozialstaatspostulat und die Demokratie.

Voigt sieht ein zentrales Problem in der Vorstellung vieler Politiker, Straftäter, geplante Anschlagsziele usw. bereits vor Eintreten der Ereignisse durch Schutzhaft, Durchsuchungen, Überwachung und andere vorverlagerte Repressionen ermitteln bzw. schützen zu können. Dies ähnelt seiner Ansicht nach der Strategie der preemptive strikes, wie sie in der nationalen Sicherheitsstrategie der USA während der Amtszeit von Präsident 
Bush vorgesehen waren. Demonstrationsverbote und Schutzzäune während großer Gipfeltreffen, wie dem der G8 in Heiligendamm, sowie das Einschleusen von V-Männern in die links- und rechtsradikale Szene, Videoüberwachung und PC-Spionage laufen Gefahr, den Kerngehalt des freiheitlichen Rechtsstaats zu unterminieren.

In Anbetracht einer vernetzten Überwachung und neuer Waffen für die Polizei, etwa den neuen nichttödlichen Waffen, die allesamt der CRC („crowd and riot control“) dienen sollen, stellt der Autor am Schluss die Frage, ob nicht der Bürger selbst von den Staatsvertretern als das eigentliche Sicherheitsrisiko angesehen wird.

\section{Julian Liese}

\section{Stephan Hensell, Die Willkür des Staa- tes: Herrschaft und Verwaltung in Ost- europa, Wiesbaden (VS Verlag für Sozi- alwissenschaften) 2009.}

Stephan Hensell leistet mit einer Kombination aus einer theoriegeleiteten Fragestellung und hervorragend recherchierten Daten zur Herrschaft und Verwaltung in Osteuropa einen originellen Beitrag zur Transformationsforschung.

Er argumentiert, dass sich in ehemals sozialistischen Staaten patrimoniale Praktiken etabliert haben, wobei traditionale und moderne Handlungslogiken nebeneinander bestehen. Staaten, die die Phase der bürgerlich-kapitalistischen Modernisierung und der rationalen Staatsbildung nicht durchlaufen haben, konnten einen patrimonialen Sozialismus entwickeln, der bis heute Bestandteil neu entstandener Machtbeziehungen ist. Hensell analysiert diese Patrimonialisierung als einen pfadabhängigen Prozess und bezeichnet die osteuropäischen Staaten als „bürokratisch-patrimonial“.

Das erste Kapitel widmet Hensell einer Kritik der Transformationsforschung, im zweiten Kapitel entwirft er eine Theorie des Staates für die untersuchte Region, im dritten Abschnitt stellt er seine methodische Umsetzung vor und entwickelt im vierten Kapitel eine strukturgeschichtliche Perspektive. Schließlich überprüft er Theorie und Methode anhand der Fallbeispiele Albanien und Georgien.
Die Transformationsforschung, so Hensell in seiner Kritik, ist erstens auf die westliche Demokratie fokussiert, widmet zweitens den postsowjetischen Republiken und den Ländern Südosteuropas vergleichsweise wenig Aufmerksamkeit und berücksichtigt drittens die Historizität der zeitgenössischen politischen Figurationen nicht ausreichend. Hensell setzt sich angesichts dieser Schwächen die Entwicklung eines Verständnisses des Staates als politische und bürokratische Ordnung zum Ziel. Dies soll durch eine Analyse der Struktureigentümlichkeit politischer Herrschaft in Osteuropa sowie die Verfolgung strukturgeschichtlicher Perspektiven wesentlicher Prozessstufen, Kontinuitäten und Brüche im Formwandel des Staates erreicht werden. Auf diese Weise sollen kausale Mechanismen, die historische Strukturen im heutigen Handeln staatlicher Akteure erkennen lassen, identifiziert werden.

Auf Grundlage der Modernisierungstheorie und der Historie des Staates wird zunächst ein theoretischer Rahmen skizziert, der auf den analytischen Kategorien Max Webers und Pierre Bourdieus aufbaut, ihre Theoreme aufeinander bezieht und zugleich reformuliert, um schließlich ein eigenes theoretisch konsistentes und empirisch plausibilisierbares Staatsverständnis zu entwickeln. Hensell kommt zu dem Schluss, dass der Staat ein von Akteurspraktiken bestimmtes Handlungsfeld ist und dem Reproduktionsmodus formal-legal gesetzter Normen unterliegt. Die Logik patrimonialer Praktiken kann als eine mögliche Praxisform im staatlichen Feld angesehen werden, in dem globale Prozesse und lokale Traditionen zu einer Vermischung von traditionalen und modernen Handlungslogiken führen.

Hensell strengt einen historischen Längsschnitt der Prozessstufen des Strukturwandels des osteuropäischen Staates an und skizziert auf diese Weise den Realtypus eines bürokratisch-patrimonialen Staates. Hierzu dokumentiert er die traditionalen Voraussetzungen, die Widersprüche des sozialistischen Staates und dessen Transformation als pfadabhängige Patrimonialisierung. Dies erfordert eine historische Schwerpunktlegung, die Hensell durchgängig gelingt. Sie untermauert seine Thesen und hilft, die
Ursachen des spezifischen politischen Wandels zu erklären.

Für seine Fallstudien hat Hensell umfangreiches empirisches Material erhoben und leistet allein hierdurch einen unverzichtbaren Beitrag zur Dokumentation der Verwaltungsgeschichte des Polizeiwesens in den osteuropäischen Staaten. Die Grundelemente patrimonialer Praktiken - Big-Men-Herrschaft, Klientelismus, ökonomische Aneignung - können anhand der von Hensell detailliert aufgearbeiteten Entwicklung der Polizeikräfte in Osteuropa sehr gut nachvollzogen werden. Er zeigt einen politisierten und militarisierten Sicherheitsapparat, der durch permanente Rotation der Polizeiämter, willkürliche Personalentscheidungen und die Etablierung einer Schmuggelökonomie gekennzeichnet ist. Hensells empirische Befunde bestätigen die These, dass die Staaten Albanien und Georgien von der Logik patrimonialer Praktiken bestimmt werden. Zwar wird die formal-rationale Perspektive der Staatsverwaltung vernachlässigt, die Konzentration auf die Mikroperspektive erlaubt es jedoch, die spezifische Einstellung der Bürger Albaniens und Georgiens zu erfassen.

Im Schlussteil verortet Hensell seine Arbeit kritisch in der aktuellen Transformationsforschung. Die große Stärke seiner Dissertation liegt in der gezielten Aufarbeitung zentraler Desiderata der Transformationsforschung sowie in der bisher vernachlässigten Erforschung der Verwaltung und des Polizeiwesens in Osteuropa. Insgesamt leistet diese wissenschaftlich fundierte, theoretisch und empirisch gut durchdachte Studie einen sehr guten Anknüpfungspunkt für die weitere Transformationsforschung.

Isabelle Gunselmann

Kurt Gritsch, Inszenierung eines gerechten Krieges? Intellektuelle, Medien und der „Kosovo-Krieg" 1999, Hildesheim (Georg-Olms-Verlag), 2010.

Im vorliegenden Band untersucht Kurt Gritsch die öffentliche Reflexion des NATO-Einsatzes im Kosovo anhand von Analysen der in Printmedien veröffentlichten Diskussionsbeiträge von „Intellektuellen“ (S. 23 ff.) sowie der politischen Berichterstattung in den Massenmedien. 
In der Debatte wurde die Frage nach der Notwendigkeit oder Legitimation einer militärischen Intervention zur Verhinderung von Gewalttaten durch Serben gestellt. Gritsch kommt zu dem Ergebnis, dass eine militärische Intervention unter Berufung auf die Verhinderung eines Genozids nicht gerechtfertigt gewesen ist. Die Rolle der Medien bewertet er als wenig neutral, sie hätten zu einer Zustimmung der Öffentlichkeit zu der militärischen Intervention beigetragen.

Nach einem Überblick über die Thematik behandelt der Autor ausführlich die Feuilletondebatten in verschiedenen deutschen Zeitungen. Er wertet die vertretenen Meinungen und Argumente quantitativ aus und kommt zu dem Schluss, dass in den untersuchten Medien Meinungsäußerungen von Gegnern und Kritikern der militärischen Intervention unterrepräsentiert waren. Auf ausgewählte Beiträge geht er intensiver ein und arbeitet Argumentationsmuster heraus. Vor allem befasst er sich mit Argumenten mit Bezug auf den Holocaust. Es folgt ein interessanter Exkurs über die Debatte um Äußerungen des Schriftstellers Peter Handke, der auf der diskursiven Ebene die Eskalation zwischen Gegnern und Befürwortern der militärischen Intervention veranschaulicht.

Erst danach legt der Autor die Vorgeschichte des Konflikts und seinen Verlauf dar: die Geschichte Jugoslawiens im 20. Jahrhundert, die Radikalisierung des Konflikts und die auslösenden Faktoren sowie den Konfliktverlauf nach der Internationalisierung. In diese Phase fallen Vermittlungsversuche zwischen den Konfliktparteien und die Stationierung von OSZE-Beobachtern, die Beteiligung der NATO und schließlich die militärische Intervention. Die Analyse der Verhandlungen führt Gritsch zu der These, die „Vermittler" hätten keine neutrale Rolle zwischen den Konfliktparteien eingenommen, sondern die serbische Regierung benachteiligt und einen Erfolg der Friedensverhandlungen sowie der OSZEMission nicht ernsthaft angestrebt. Anschließend werden die Auswirkungen der militärischen Intervention beleuchtet, dabei geht der Autor auf Berichte ein, die seine These, die Legitimation des militärischen Einsatzes als „humanitäre Intervention“ habe lediglich als Vorwand gedient, unterstützen sollen.
Als westliche „traditionalistische Interpretation“ der Geschehnisse bezeichnet er die Sichtweise der Befürworter einer militärischen Intervention und findet sie auch in geschichtswissenschaftlichen Veröffentlichungen. Der Autor kommt in seiner Kritik zu dem Ergebnis, der Traditionalismus diene „damit schlussendlich weniger der wissenschaftlichen Erkenntnissuche denn der postumen Rechtfertigung der westlichen Politik“ (S. 281). Schließlich stellt Gritsch die Beiträge der Friedensforschung - Alternativvorschläge zur Behandlung des Konflikts - vor.

In einem weiteren Teil untersucht der Autor die Motive der intervenierenden NATO-Staaten anhand einer Analyse der Interessen der USA, der EU und Deutschlands im Kosovo. Unter anderem wirft er den politisch Verantwortlichen in Deutschland vor, insbesondere die militärische Emanzipation der Bundeswehr im Auge gehabt zu haben, wofür auch die deutsche Öffentlichkeit gewonnen werden sollte. Neben den europäischen Akteuren und den USA, die der Autor als zumindest teilweise machtpolitisch motiviert beschreibt, wird die NATO als unabhängige Kraft dargestellt, die an Einfluss, auch gegenüber der UNO, gewinnen und nach dem Ende des Kalten Krieges in ihrer Existenz legitimiert werden wollte. Bisweilen gerät der Ton populistisch, etwa wenn Gritsch schreibt, die NATO wolle nicht durch leere Drohungen, als ,impotent“ gelten. Hier stellt er einen Zusammenhang mit der Clinton-Lewinsky-Affäre her. Des Weiteren führt er neoliberale, geostrategische, geopolitische und militärische Interessen der beteiligten Parteien als Motive für einen Militäreinsatz an. Bei der Analyse der Interessen bedient sich der Autor, wenn keine sicheren Quellen verfügbar sind, nach eigenen Angaben „der ineinander greifenden Prinzipien Skepsis und Plausibilität“ (S. 18). Neben der Darstellung der ökonomischen Folgen des Krieges im Kosovo geht Gritsch auch allgemein auf die „neoliberale Weltwirtschaft als Kriegsmotor" ein (S. 335 ff.). Der Abschnitt verrät seine kritische Einstellung gegenüber der Politik von Weltbank und Internationalem Währungsfonds.

Kurz behandelt Gritsch die Frage der Legitimation von „humanitären “ Interventionen aus philosophischer, historischer und völkerrechtlicher Perspektive und kommt zu dem Schluss, dass humanitäre Interventionen durchaus moralisch gerechtfertigt sein können. Er wendet die Merkmale einer humanitären Intervention auf den Kosovo-Fall an und kommt dabei zu dem Ergebnis, dort seien die Bedingungen für ein solches legitimes Eingreifen nicht gegeben gewesen - stattdessen müsse von einem Angriffskrieg gesprochen werden (S. 388).

Zuletzt befasst sich Gritsch unter der Überschrift „Linke und Krieg“ vor allem mit der Rolle von Spitzenpolitikern der Partei Bündnis90/Die Grünen, die als Koalitionspartner der SPD die militärische Intervention befürworteten. Speziell geht der Autor auf den damaligen Außenminister Joschka Fischer ein, dessen „Sinneswandel“ vom Kriegsgegner zum Kriegsbefürworter er als unglaubwürdig ansieht und Fischer stattdessen vorwirft, aus reinem Machtstreben systematisch Partei- und Wählertäuschung betrieben zu haben.

Insgesamt ist das Buch sowohl für Leserinnen und Leser mit geringen Kenntnissen über das Geschehen empfehlenswert als auch für diejenigen, die die Debatte um den Kosovo-Einsatz in den Medien verfolgt haben; für Erstere empfiehlt es sich, mit Kapitel 8, in dem der historische Hintergrund erläutert wird, zu beginnen. Der Autor vertritt seine Interpretationen sehr dezidiert; er nimmt eine sehr interventionskritische Haltung ein und argumentiert insgesamt aus einer „linken“ Perspektive. Die Veröffentlichung bietet auch über den Kosovo-Krieg hinaus Denkanstöße zu den Themen NATO, Interessenspolitik, humanitäre Intervention und ihre Darstellung in westlichen Medien.

Sabine Undorf

\section{Solveig Richter, Zur Effektivität externer Demokratisierung. Die OSZE in Südost- europa als Partner, Mahner, Besserwis- ser?, Baden-Baden (Nomos) 2009.}

Die Debatte um positive wie negative Aspekte der externen Demokratisierung ist nicht neu, auch die Frage nach der Effektivität von Eingriffen in die staatliche Souveränität ist häufig gestellt worden. Oftmals sind die Überlegungen hierzu wenig ertragreich gewesen, da keine ge- 
genstandsangemessene und differenzierte Betrachtung vorgenommen worden ist.

Im Kontext der komplexen Transformationsprozesse in Südosteuropa untersucht Solveig Richter die Wirkung der OSZEMissionen anhand der Fälle Bosnien und Herzegowina, Kroatien und Serbien. Die unterschiedlichen beteiligen Akteure, ihre Ziele und die verwendeten Instrumente finden ebenso Beachtung wie die historischen und sozialen Hintergründe in den jeweiligen Ländern. Ziel der Analyse ist es erklärtermaßen, die Strategien für die jeweiligen Transformationsphasen auszumachen und die Effektivität der Demokratisierungsmaßnahmen zu untersuchen. Zudem soll sich eine Symbiose aus dem „Was“ der Transformationsforschung und dem „Wie“ der Theorie der Internationalen Beziehungen einstellen (S. 31).

Gleich zu Beginn werden die Fragen nach Wirkungsweise, Legitimität und Erfolgsmöglichkeit solcher Demokratisierungsvorhaben gestellt. Die Bezeichnung der Demokratie als universale Staatsform, als „Wunderwaffe” (S. 44) sieht Richter kritisch und verweist auf negative Auswirkungen von Demokratisierung, wie beispielsweise die Destabilisierung eines Landes oder der Etablierung westlicher Vorherrschaft. Die Kategorien für ihr Untersuchungsdesign gewinnt sie aus Idealtypen und bezeichnet die unterschiedlichen Ausprägungen der Interaktionen mit den Begriffen Partner, Mahner/Besserwisser und Versager. Die mögliche Effektivität der Maßnahmen wird ebenfalls in unterschiedliche Kategorien gefasst: effektiv (sowohl positiver Einfluss als auch erreichte Ziele), subeffektiv (nur einer der beiden Faktoren) und ineffektiv (weder noch). Als Referenz für die Effektivitätsanalyse dienen der Autorin der „hypothetische Zustand” und das „kollektive Optimum” (S. 56): eine 'Was wäre, Wenn'-Situation ohne Intervention gegenüber Annahmen eines Idealzustands im Land. Ferner wird der Leser/die Leserin durch eine Darstellung der historischen und strukturellen Hintergründe der OSZE und Südosteuropa in die Gesamtproblematik eingeführt. Den Ausgangspunkt bildet die „Hilflosigkeit der internationalen Gemeinschaft” (S. 115) angesichts der ökonomischen Krisen, Unabhängigkeitsbestrebungen und akuten Konflikten in den untersuchten Ländern.

In ihrer Arbeit verfährt Richter nach einem konstanten Muster. Zunächst schildert sie Verlauf, Schlüsselakteure und Schlüsselbereiche der Demokratisierung, auch das jeweilige Mandat und dessen Ziele und Instrumente werden analysiert. So sei das Mandat beispielsweise in Bosnien und Herzegowina, nicht zuletzt aufgrund des Dayton-Abkommens über Transformation und internationale Supervision, „speziell“ (S. 146) gewesen. Die OSZE hatte hier die Möglichkeit, Sanktionen zu verhängen und ihren Forderungen Nachdruck zu verleihen. Bei der von Richter vorgenommenen Effektivitätsanalyse muss die Organisation dennoch Federn lassen: In einigen Bereichen, wie dem Schutz der Menschenrechte, hat sie Verbesserungen erzielt, in anderen, wie der angestrebten Bildungsreform, nahezu versagt, ist sie „ineffektiv“ gewesen.

Dieses ambivalente, differenzierte Bild des Wirkens der OSZE und seiner Effektivität zieht sich recht kontinuierlich durch die gesamten Analysen. Bei der Mission in Kroatien sei beispielsweise die Demokratisierung gar nicht erst durch Organisationen wie die OSZE vorangebracht worden, sondern vielmehr durch den internen Prozess der nationalen Unabhängigkeitsbestrebungen. Die OSZE sei hier auch nur dann effektiv gewesen, wenn es windows of opportunity oder Koalitionen mit anderen internationalen Akteuren gegeben habe. In Serbien seien Verbesserungen, wenn überhaupt, dann nur oberflächlich erreicht worden. Auch bestanden große Diskrepanzen bezüglich Südserbien und Belgrad. Die OSZE war hier laut Richter zu schwerfällig und reagierte nicht auf wachsende Ansprüche bei steigendem Demokratisierungsgrad.

Bei der abschließenden vergleichenden Analyse arbeitet Richter Gemeinsamkeiten und Muster heraus. So hält sie beispielsweise fest, dass der Wechsel von autokratischen Systemen zu neuen Reformbündnissen in allen drei untersuchten Fällen im Jahr 2000 stattfand. Auch bei vielen Grundproblemen zeigten sich Ähnlichkeiten: das Problem der Flüchtlingsrückkehr, defizitäre Wahlsysteme, Mängel bei Medien, Menschenrechtsschutz und der Rechtsstaatlichkeit. Richter bescheinigt der OSZE einen insge- samt positiven Einfluss auf die Transformationsprozesse, schränkt aber ein, dass die Ziele in den einzelnen Fällen zwar ähnlich gewesen seien, die Strategien und Instrumente zu ihrer Erreichung sich jedoch deutlich unterschieden hätten. Keine der Demokratisierungsstrategien sei als durchgängig effektiv zu bezeichnen. Sie hält darüber hinaus fest, dass internationale Akteure in einem fremden Land per se bereits ein „Defekt der Demokratie“ (S. 371) seien und externe Demokratisierung mit Vorsicht und Bedacht eingesetzt werden sollte.

Richter bietet in ihrer Dissertation eine sehr gründlich recherchierte und sehr umfassende Analyse der Demokratisierungsbemühungen der OSZE in Südosteuropa. Ihre Einschätzung, dass diese Überlegungen auf andere Regionen übertragbar sind, ist durchaus vertretbar. Sie erhebt keinen moralischen Zeigefinger, sondern geht strikt empirisch vor, was dem wissenschaftlichen Ertrag der Arbeit sehr dienlich ist.

Cosima Zentara

\section{Merle Vetterlein, Konfliktregulierung durch power-sharing-Modelle: das Fall- beispiel der Republik Makedonien. De- mokratie, Sicherheit, Frieden Band 196, Baden-Baden (Nomos) 2010.}

Die Arbeit von Merle Vetterlein betrachtet die Wirksamkeit von Power-sharing-Modellen als konfliktregulierende Maßnahmen am Beispiel der Republik Makedonien. Jahrelang hatte das Volk der Makedonier für seine Anerkennung als Nation und somit für sein Anrecht auf einen eigenen Staat gekämpft, bis es 1991 nach dem Zerfall Jugoslawiens einen unabhängigen Staat erhielt. Doch seitdem herrschte stets ein Konflikt zwischen der makedonischen Titularnation und der albanischen Minderheit, die etwas mehr als 20 Prozent der Gesamtbevölkerung ausmacht. Die albanische Minderheit forderte eine stärkere Einbindung in die Politik des Landes und die Anerkennung ihrer Kultur. 2001 kam es zu einem gewaltsamen Ausbruch des Konflikts, der etwa 400 Opfer forderte. Dieser Konflikt konnte im selben Jahr mit dem Rahmenabkommen von Ohrid beigelegt werden.

In einem Theorieteil stellt Vetterlein zunächst die Cleavage-Theorie von Lip- 
set und Rokan und ein Analysemodell von Knutsen und Scarborough vor. Die Cleavage-Theorie unterscheidet zwischen sogenannten super-imposed- und cross-cutting-cleavages. Beide bezeichnen Konfliktlinien: erstere beschreiben parallel verlaufende, die Gesellschaft polarisierende Linien, letztere sich überschneidende und die Konfliktparteien übergreifende Linien. In der Republik Makedonien finden sich viele superimposed-cleavages: Die Kluft zwischen den ethnischen Makedoniern und den makedonischen Albanern ist sowohl in den Bereichen der Ethnie, der Politik, der Geografie, der Sprache, der (Sozio-)Ökonomie, der Bildungspolitik als auch der Religion erkennbar. Das Parteiensystem institutionalisiert die cleavages und spiegelt diese wider.

Zur Erfassung der Akteursstrukturen in dem untersuchten Land führt Vetterlein den Begriff der „Eliten“ ein. Er umfasst sowohl demokratisch legitimierte Eliten, wie z.B. Parteien als auch die Eliten illegaler und informeller Einrichtungen. Auch deren Interessen müssen bei der politischen Entscheidungsfindung berücksichtigt werden, selbst wenn sie für die Öffentlichkeit nicht ohne Weiteres sichtbar werden, wie dies im Falle organisierter Kriminalität, Klientelismus oder von Korruption gegeben ist.

Vetterlein stellt fest, dass die albanischen Makedonier sich unterdrückt fühlen und für ihre Anerkennung als zweite staatstragende Nation kämpfen. Sie fordern die Anerkennung des Albanischen als zweite Amtssprache und eine größere Repräsentanz in Armee, Polizei, Justiz und Verwaltung. Des Weiteren wollen sie ihr Recht auf eine albanischsprachige Universität verwirklicht sehen. Diese Forderungen sind auch auf die schwachen Institutionen des politischen Systems zurückzuführen: Das Wahlsystem mit Mehrheitswahlrecht führte zu einer Überrepräsentation der slawischen und marginalisierte die albanischen Parteien im Parlament, was die ethnische Segregation zementierte und alte Eliten aus der Zeit Jugoslawiens begünstigte. Die Oppositionsrolle war in der Folge eher ethnisch als politisch geprägt. Die defizitäre staatliche Verwaltungsstruktur bewirkte überdies eine Überzentralisierung. Dies verfestigte die Verbindung zwischen politischer Macht und dem
Zugang zu wirtschaftlichen Ressourcen und marginalisierte die ländlichen Gegenden, in denen aufgrund der ethnisch unterschiedlichen Siedlungsstruktur zumeist Albaner lebten. Nepotismus und Korruption konnten in den staatlichen Institutionen und dem Parteiensystem Überhand gewinnen.

Die Arbeit legt den Schwerpunkt auf die Analyse der Konfliktstruktur und führt die verschiedenen Hintergründe des Konflikts auf die Sozialstruktur des Landes zurück: Der vorangegangene Kosovo-Krieg hatte 335.000 albanische Flüchtlinge in die Republik Makedonien geführt. Die makedonische Titularnation fühlte sich durch die Albaner, die nun ein Drittel der Gesamtbevölkerung ausmachten, bedroht. Vetterlein sieht die Ursachen des Konflikts in der Befürchtung, dass die Flüchtlinge den Kosovokrieg in Makedonien weiterführen könnten, jedoch auch im inneralbanischen Machtstreit und im Vorhandensein Organisierter Kriminalität. Am 22.1.2001 führte ein Granatenanschlag bei Tetovo, der den Tod eines Polizisten zur Folge hatte, zu einem gewaltsamen Konfliktausbruch. Die internationale Gemeinschaft griff schnell ein und bereitete den Weg für das Rahmenabkommen von Ohrid. Das Rahmenabkommen strebte einen Interessenausgleich zwischen Albanern und Makedoniern sowie Demokratie, Dezentralisierung und Gewaltfreiheit bei der Durchsetzung politischer Ziele an, unter Bewahrung des multiethnischen Charakters und der territorialen Integrität des Landes. Power-sharing-Ansätze des Friedensvertrags sahen Vetorechte für Minderheiten vor und Sonderrechte beispielsweise bei den Minderheitensprachen, was zur Stärkung von Gruppenrechten und letztendlich zu zwei (inoffiziellen) Verwaltungssprachen führte.

Bei der Implementierung traten die Mängel des Abkommens zu Tage: Die Vertragsparteien - die zwei größten ethnisch-makedonischen und albanischen Parteien - vereinbarten die stärkere Einbindung der Albaner in die Regierung. Die albanische UCK, die zuvor gewaltsame Anschläge verübt hatte, wurde nicht beteiligt. Das Parlament wurde in seiner Bedeutung herabgestuft, insofern es Verfassungsänderungen ohne jegliches Mitspracherecht hinnehmen musste. Die Koalitionsbildung zwischen albanischer und ethnisch-makedonischer Partei, wie sie seit der Unabhängigkeit üblich war, sollte weiterhin auf der Basis von Freiwilligkeit vorgenommen werden. Dies führte dazu, dass keine power-sharing-Exekutive errichtet wurde. Ein Eingehen auf die Bedrohungsängste der Titularnation war nicht möglich. Zu enge zeitliche Vorgaben und eine voranschreitende Marginalisierung kleinerer ethnischer Minderheitengruppen waren weitere Folgen. Viele Sonderrechte galten nur für die albanische Minderheit. Als Komponenten der consociational democracy nennt die Autorin eine „positive Diskriminierung“ an Universitäten und eine Proporzregel für den öffentlichen Dienst.

Trotz Stagnation der interethnischen Annäherung in einigen Bereichen muss das interethnische Zusammenleben seit 2001 aber insgesamt als erfolgreich gewertet werden, da es gewaltfrei geblieben ist. Vetterlein stellt abschließend fest, dass „gesellschaftliche und interethnische Segregation nicht zwangsläufig zu gewaltsamen Auseinandersetzungen führen muss“ (S.239). Der mögliche EU-Beitritt könne zudem als Druckmittel genutzt werden, um der Destabilisierung eines Landes mit politisch schwachen Entscheidungsträgern entgegenzuwirken.

Die gelungene Konfliktanalyse vermittelt dem Leser ein gutes Verständnis der Situation in der Republik Makedonien. Die Dissertation ist bereits deshalb eine lohnende Lektüre, weil sie die sonst nur spärlich vorhandenen Informationen über die Konflikte in dem Land zusammenträgt. Die Bewertung der Konfliktregulierung fällt etwas knapp im Verhältnis zur Konfliktanalyse aus, doch ohne eben diese ausführliche Konfliktanalyse wären die Auswirkungen des Abkommens von Ohrid nur schwer verständlich. Die Republik Makedonien, den meisten nur als ein friedlicher ehemaliger Staat Jugoslawiens bekannt, hat seinen Nations- und Staatsbildungsprozess gerade erst abgeschlossen und muss sich vor dem schwierigen Hintergrund ethnischer Auseinandersetzungen noch festigen. Die Arbeit gibt neben der Darstellung des Konflikts auch einen guten theoretischen Überblick über die power-sharing-Modelle, die im Rahmenabkommen von Ohrid zum Einsatz gekommen sind.

Inga Zimmermann 
Rainer Huhle (Hrsg.), Human Rights and History: A Challenge for Education, Berlin (Foundation „Remembrance, Responsibility and Future") 2010.

"Human Rights and History" may in fact be a misleading title for this rich collection of papers on human rights, as it suggests a focus on the past only. However, the appeal of this work on human rights and historic learning processes is precisely that it bridges the past and the present by enquiring into the "relationship between historical awareness and current engagement" (p. 5). The volume contains papers discussed at the international conference "Rights that make us Human Beings" which gathered in Nuremberg in 2008 at the historic location of the Nuremberg District Court, where the Nuremberg trials were held from 1945 to 1949. Throughout the volume the great importance of the past - especially the Nazi horrors and the international reactions to them after 1945 - is prominent, but considerations on present challenges and the ongoing struggle for human rights are also included. Although the single contributions do not always manage to relate past and present, the volume when seen holistically - connects these in a very perceptive way, giving the reader a valuable feel for the interrelatedness of concrete experiences of injustice and the creation and enforcement of universal human rights as an ongoing struggle.

The opening presents a moving interview with Stéphane Hessel, who survived three Nazi concentration camps and has since been working on behalf of human rights. This is followed by several papers which enquire into the historical origin of human rights and concepts such as crimes against humanity and genocide, particularly by analyzing the drafting of the Universal Declaration and the Nuremberg Trials.

For instance, Johannes Morsink's paper attempts to use the history of the Universal Declaration as proof for the general universal nature of human rights. Morsink stresses the human capacity to feel repulsed by injustice as a precondition and basis for the universality of human rights. In this respect he addresses the central question of whether human rights are "man-made" (p. 26), thus dependent on cultural norms, or naturally inherent to all humans. His answer is clearly that they are inherent and accessible by all human beings due to the moral conscience they share. Essentially, his reasoning draws on the process of drafting the Universal Declaration, which was based on a shared revulsion by the horrors of Nazi Germany: Delegates from across the globe, from 56 nations, were all so deeply repulsed by these crimes that they reached a consensus (with ten abstentions, but no votes opposed to the adoption of the Declaration). By tracing back the discussions and coalitions in drafting the declaration, Morsink attempts to show that the critique of a Western bias in the Universal Declaration is misplaced. His argument, although revealing interesting dynamics at play in the drafting, is, however, not fully convincing, especially because it downplays the ongoing conflict of prominent human rights conceptions and Islamic understandings of Shari'a law. Especially in a volume on the history of human rights - a relatively young history - the question of whether human rights were "discovered" or "invented" could have been discussed in greater depth. If all human beings share a moral sense and revulsion against injustice, how can it be that for centuries some social groups have been systematically dehumanized and discriminated against? This paper shows that focusing on a part of human rights history and universalizing from this entails the danger of fading out ongoing current and boundary problems. Yet, while the beginning of the volume might at times commit this mistake, the following papers help bridging past and present, while more openly addressing controversies. "As such, human rights education must avoid presenting human rights as timeless, eternal, and uncontested" (p. 179), it says in a later contribution, and on the whole the volume lives up to this demand.

This is also thanks to the contributions in the second section which look at the formulation and the still ongoing evolution of particular human rights, such as the freedom of expression and the right to asylum. These papers begin to close the gap between history and current human rights issues, for example when discussing different angles of discrimination or the limits of freedom of expression, pointing to some profound problems that remain to be solved.

An especially powerful piece in this respect is Aidan McQuade's paper on new forms of slavery in the 21st century. Vitally, slavery is not meant metaphorically here: McQuade stresses that according to definitions of the term slavery by the UN or the International Labor Organization (ILO), there are still a minimum of 12.3 million people in slavery today. McQuade points to current instances of slavery such as debt bondage, trafficking and child labour, illustrating his argument with appalling case examples. Yet the contribution goes far beyond a mere descriptive account. Not only does the author vividly depict different angles of the current problem such as the cruel connection of slavery and war, but he also points to possible solutions. McQuade's contribution manages to combine several dimensions of the problem of slavery - economic, political and social - as well as several levels of causes and solutions from the vantage points of different actors. Importantly, McQuade argues that "all countries in the world are affected by slavery to some degree" (p. 121) and he sees room for action on all fronts. He criticizes the South Asian countries in which bonded labor is tolerated, but also the EU for failing to adequately implement their Convention on Action against Trafficking on Human Beings. One of the central solutions suggested by McQuade is universal primary education for all children, which could reduce child labor as well as the vulnerability to future enslavement. However, instead of presenting national governments and international organizations as the only potential eradicators of slavery, he asks for combined efforts of governments, business and civil society. Vitally, McQuade's contribution emphasises that human rights protection is an on-going struggle. When sketching the mutation of forms of slavery over time in response to abolition campaigns, he urges for efforts to constantly reassess working conditions against a consistent standard in order to protect human rights lastingly.

The rest of the volume offers other similarly insightful contributions on the struggle and learning processes in 
relation to human rights issues. One section is dedicated to specific case studies: the Helsinki Movement in Central and Eastern Europe as well as the Latin American case of Colombia where particular difficulties of enforcing human rights in a society characterized by grave injustices and persistent armed conflict are faced.

The underlying theme of the volume also visible in the subheading A Challenge for Education - is most explicitly, but only relatively briefly, addressed in the last section. Unfortunately, this focuses on European contexts only and fails to consider the even bigger challenge of human rights education in developing countries. In essence, the contributions revolve around the question of how much history is necessary and useful in human rights education. Emphatically, Scherr warns that the history of the Holocaust "does not automatically confer an appreciation for human rights as fundamental principles" (p. 175). The contributions reflectively look at target audiences and respective methods of teaching. They appreciate some diversity in the student body - which is comprised not only of academics but largely of children and adolescents, some of which have immigrant backgrounds. Generally, they call for better targeted teaching strategies, including issues of personal significance (such as the history of migration in immigrant societies), which help explore political, legal and moral facets of human rights. Besides history, which is, according to the authors, still a crucial element in the learning process, other vital parts of human rights education are, "memory one's personal connection to the past" (p. 171), as well as, an intense "democratic engagement from the educators" (p. 174). Overall, the prevailing argument in these contributions is in favor of drafting teaching programs which "bridge the past and the present" (p. 166). Walking this line between historic and current issues is certainly a challenge for educators, yet this volume gives some proof that it can be done successfully.

Wiebke Marie Junk

\section{Sicherheit in Südasien}

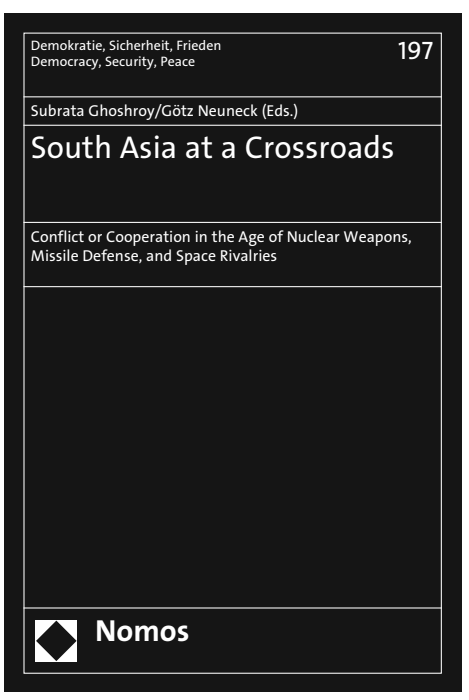

South Asia at a Crossroads

Conflict or Cooperation in the Age of Nuclear Weapons, Missile Defense, and Space Rivalries Herausgegeben von Subrata Ghoshroy und Götz Neuneck 2010, 330 S., brosch., 69,- $€$ ISBN 978-3-8329-5398-0

(Demokratie, Sicherheit, Frieden, Bd. 197)

Der Sammelband enthält aufschlussreiche Beiträge zu einer Reihe aktueller Fragen zur Sicherheit in Südasien aus globaler Sicht. Das Buch zeichnet sich dadurch aus, dass es schwierige Themen an der Schnittstelle von Wissenschaft, Technologie und Politik allgemein verständlich darstellt. Es führt Aufsätze von Wissenschaftlern, Diplomaten, Abgeordneten und hochrangigen Politikberatern aus Asien, Amerika und Europa zusammen und behandelt Fragen, die sowohl für die globale Sicherheit als auch für die Region von Bedeutung sind, vor allem für die benachbarten Atommächte Indien und Pakistan, die im Fokus des Bandes stehen.

Behandelt werden u.a. Raketenabwehr, satellitengestützte Überwachung zur Verbesserung der strategischen Sicherheit und Weltraumrüstung. Auch der beginnende „Wettlauf ins All“ in Asien wird unter die Lupe genommen. Des Weiteren befasst sich der Band mit der Zukunft der nuklearen Rüstungskontrolle, Abrüstung und vor allem mit dem Nichtverbreitungsvertrag.

Der Band enthält kritische Auseinandersetzungen mit dem kürzlich abgeschlossenen Nuklearabkommen zwischen den USA und Indien sowie Beiträge zur Energiesicherheit Pakistans und Indiens. Wie der Titel bereits andeutet, sondiert das Buch Möglichkeiten der regionalen Kooperation in vielschichtigen und konfliktträchtigen Fragen.

"The book has a regional focus but a global message. It is simply excellent and particularly profound." Prof. Dr. Kai-Uwe Schrogl, ZLW 3/10

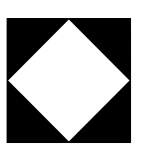

\section{Nomos}

Bitte bestellen Sie im Buchhandel oder

versandkostenfrei unter $\checkmark$ www.nomos-shop.de 